\title{
Measuring Incident Light on Grape Clusters Using Photosensitive Paper and Image Analysis
}

\author{
Steven F. Price and Marc L. Schuette \\ Department of Horticulture, Oregon State University, Corvallis, OR 97330 \\ Elizabeth Tassie \\ Charles Sturt University, Riverina, Wagga Wagga, New South Wales, Australia \\ Additional index words. Vitis vinifera, grapevine, canopy management
}

\begin{abstract}
Digital imaging and analysis was used to quantify and characterize the light exposure patterns of photosensitive paper tubes placed in representative cluster positions in two grape (Vitis vinifera L.) canopies: a minimally pruned and a vertically trained canopy. Blue pixel values of the captured images had a negative correlation with the log of irradiance from an integrating quantum sensor $\left(r^{2}=\mathbf{0 . 9 3 0 8}\right)$. The spectral response of the photosensitive paper was not measured. Histograms of incident light distribution on individual paper tubes were developed using imaging software. Histograms were able to quantify the distribution of incident light on individual tubes and were clearly related to the tube's exposure in the canopy. Average population curves of pixel light distribution of 20 tubes in each canopy were able to differentiate the typical cluster light environment in the two canopies. Tubes in the minimally pruned canopy had a larger proportion of their surface exposed to irradiances $>50 \mu \mathrm{mol} \cdot \mathrm{s}^{-1} \cdot \mathrm{m}^{-2}$ and $65 \%$ higher average irradiance than the vertical canopy. Image analysis of photosensitive paper appears to be a workable method to record the distribution ofincident light in plant canopies and may have utility in a range of ecological studies.
\end{abstract}

The light environment of grape clusters can have pronounced effects on grape physiological processes and fruit and wine composition. Grape acidity, aroma, flavor, color, and phenolic composition are influenced by light. We have been investigating the effects of sunlight on the phenolic composition of grape skin and have found that light exposure greatly increases flavonol content (Price et al., 1992, 1994). Only grape skin directly exposed to full sunlight appeared to show this response. As a result, significant variation can exist in flavonol content between shaded and sun-exposed sides of the same cluster, between clusters with different exposures on the same vine, and between typical clusters from different canopy systems. As one of the first steps in developing a model to explain the variation in flavonol content of grapes, we needed a method to quantify and characterize the incident solar radiation on the entire cluster surface, for individual clusters and representative clusters in canopy systems where light environments may vary.

Several approaches have been used to describe canopy effects on the light environment of grapes. The most common arc descriptions of whole-vine structural parameters, such as leaf area indicts, leaf layer number, and number of shoots or pruning weights per unit length of canopy affecting the light environment of clusters (Shaulis et al., 1966; Smart, 1985). Point quadrat analysis has been used to describe several parameters, including canopy gaps, leaf layer number, and percent fruit exposure relating to cluster light exposure (Smart, 1982; Reynolds and Wardle, 1989a). Fisheye photography has been used to describe light transmission through canopies (Reynolds and Wardle, 1989a) and measures of sunflecks have been used to indicate light penetration into a canopy (Smart, 1988). While all these methods can be used to estimate changes in the canopy light environment on a whole-plant level, none

Received for publication 20 June 1994. Accepted for publication 7 Nov. 1994. Oregon Agricultural Experiment Station paper no. 10,219. This research was supported in part by the Oregon Wine Advisory Board. The cost of publishing this paper was defrayed in part by the payment of page charges. Under postal regulations, this paper therefore must be hereby marked advertisement solely to indicate this fact. describes the incident light on an individual cluster.

Light sensors of various types have been used to describe canopy effects on light quantity and quality on a whole-plant level and measure incident light on clusters (Reynolds and Wardle, 1989b). Smart (1988) used a spot quantum meter to describe the photosynthetic photon flux density incident on individual clusters. He found intensive sampling was required under sunny conditions due to variation in sunflecks and sensor angle. To get acceptable readings, it was necessary to avoid sun flecks or take readings on cloudy days. Because of these same factors, we have been unable to obtain repeatable light readings for individual clusters using a spot quantum sensor.

Long-term placement of multiple sensors in plant canopies can eliminate problems with sunfleck variation (Reifsnyder et al., 1971), but problems with sensor placement and the geometry of the sensor and incident light can still be significant. Gutschick et al. (1985) used large arrays of nonfiltered photodiodes to measure light incident on individual leaves. Multiple sensors were attached directly to the leaves and histograms of irradiance were used to describe variation of incident light on leaves with different angles and canopy positions. This method provides significantly more information than single-sensor measurements, but equipment requirements arc substantial.

In response to these problems, we developed a system for describing cluster light environment using photosensitive paper and computer image analysis. Photosensitive ozalid papers have been used to quantify the light environment in numerous ecological studies (Emmingham and Waring, 1973; Friend, 1969). They were primarily used as a simple integrator. Stacks of paper were exposed to light and the number of colored layers were correlated to light meter readings. In our study, computer image analysis was used to describe the patterns of light exposure on individual sun exposed papers. The captured images provide detailed information on the distribution of light incident on individual clusters. The objective of this study was to determine if photosensitive paper and image analysis could describe differences in incident light on individual clusters and if this information could then be used to 


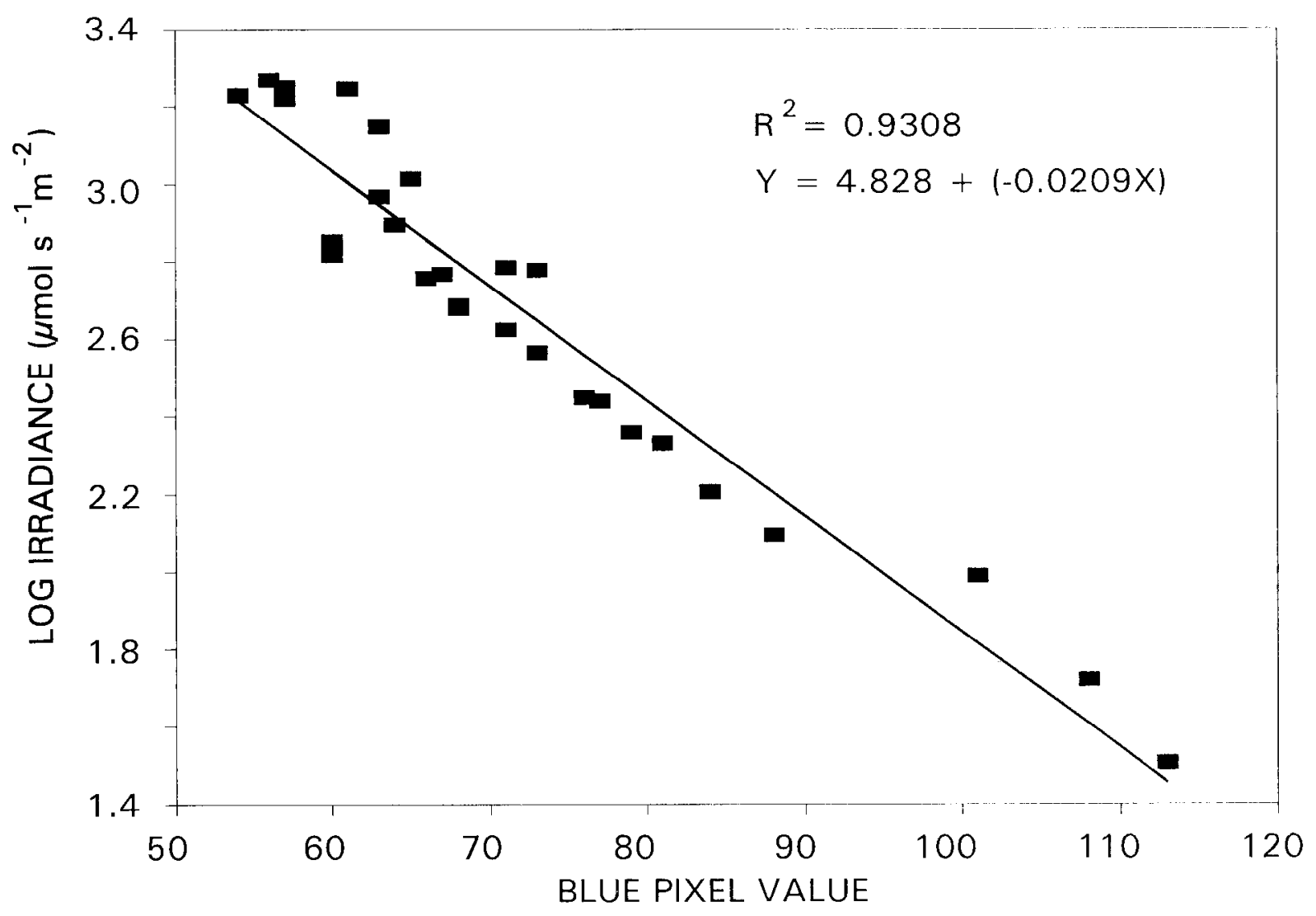

Fig. 1. Calibration curve for Sunprint photosensitive paper. Light levels were recorded with a cosine corrected quantum meter

describe the average cluster light environment in canopy management studies.

\section{Materials and Methods}

Grape canopies. Two, 20-vine sections of 12-year-old 'Cabernet Sauvignon' from an existing minimal-pruning experiment were selected based on apparent differences in cluster exposure. One section was a vertical canopy with shoots positioned upward; the other was a minimally pruned canopy with no shoot positioning (Clingleffer, 1984). The vertical canopy was cane pruned annually and the sides and top were hedged once at shatter. The minimally pruned canopy had no dormant pruning for 3 years. Shoots hanging below the support wire were pruned off at bloom. The canopy consisted of short shoots with clusters located toward the outside of the canopy. Reduced shoot growth and exterior cluster positions are typical vine responses to minimal pruning (Clingleffer, 1984). The vertically trained vines were vigorous vines (pruning weights the previous season averaged $1.7 \mathrm{~kg} \cdot \mathrm{m}^{-1}$ of canopy), with long shoots and clusters generally located in the lower interior of the canopy. The row orientation in the plot was north to south.

Photosensitive paper. Sheets of photosensitive paper were from Sunprint Kit (Univ. of California, Berkeley), an educational science toy. When exposed to sunlight, the paper becomes blue. The wavelength response range for the photosensitive paper used in this study was not available from the manufacturer, but ozalid papers used in other studies were most responsive in the violet and ultraviolet wavelengths (Emingham and Waring, 1973). Tests of Sunprint paper in this study with a wratten \#12 filter suggest that paper was not affected by wavelengths $>500 \mathrm{~nm}$ (data not shown). Sensitivity to shorter wavelengths may be an advantage for our work with flavonols since blue and ultraviolet UV light have been shown to increase flavonol synthesis in plants (Brodenfeldt and Mohr, 1988 Stafford, 1990).

Calibration. Twenty-nine $50 \times 50-\mathrm{mm}$ sheets of photosensitive paper were exposed to varying light intensities under full sun, cloud cover, and in various plant canopies for $5 \mathrm{~min}$. Light exposure levels for calibration were deliberately selected to obtain a complete range from full sun to deep shade. Light levels were recorded on an integrating quantum sensor (LI-188B; LI-COR, Lincoln. Neb.). The paper and sensor were placid adjacent to each other on a rigid board to ensure that the angle of light interception was the same for both. Exposed papers were developed in running water for $2 \mathrm{~min}$ and air dried for 3 days before image capture and analysis.

Cluster exposure analysis. Forty $100 \times 100-\mathrm{mm}$ sheets of photosensitive paper were taped into tubes $(31.8 \mathrm{~mm}$ in diameter), reactive side out, in a darkroom under a safe light with a wratten series OA safe light filter and kept in a dark box until placed in a canopy. Twenty paper tubes were placed in each canopy type in randomly selected cluster positions, one per vine. Each cluster on a vine was assigned a number, and a random number generator was used to select the cluster position for analysis. Paper tubes were placed near the selected clusters in a similar light environment and orientation (grape clusters are roughly cylindrical in shape). The paper tubes were held in place with an alligator clip on a flexible wire. Tubes were put out in pairs, one in each canopy. at 1-min intervals during the course of the experiment. The experiment was conducted under clear skies at midday in early September. After a 5-min exposure, tubes were placed in a dark bug. Later they were opened, flattened, developed, and dried as described above.

Image capture and analysis. The images of the exposed sheets 


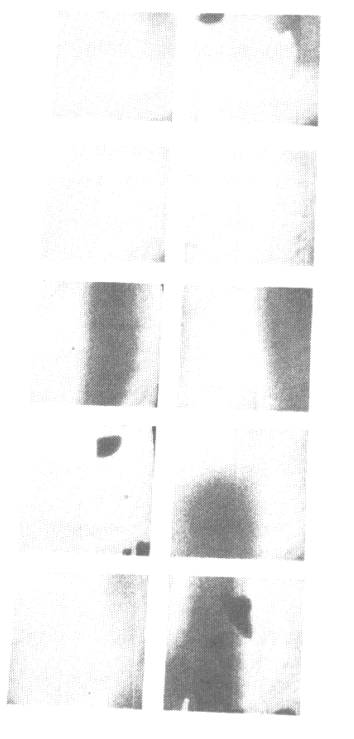

UPRIGHT TRELLIS

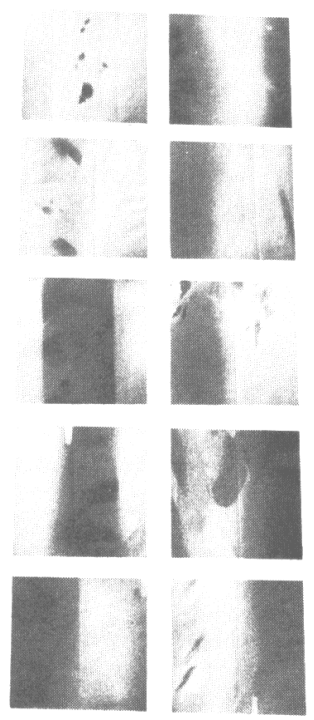

MINIMUM PRUNING
Fig. 2. Photosensitive papers from two grape canopies: an upright vertical canopy with clusters at the base of the canopy and a minimally pruned canopy with clusters on the periphery of the canopy. Papers were rolled into a cylindrical shape and placed in random cluster locations. Darker areas are more sun exposed. Dark spots are from sun flecks.

were captured with a video camera on a light stand with two 60Watt incandescent bulbs. Lighting conditions and camera settings were kept constant for exposed sheets from the canopies and calibration set. Video output was to an image capture, digitizing, and display adapter with $512 \times 512$ pixel resolution and 256 levels of intensity for each of the red, green, and blue pixel components [(Truevision (TARGA-24); AT\&T Indianapolis]. The digital data from the captured image were analyzed using an in-house program that gives minimum, maximum, and average pixel intensity values for red, green, and blue for the entire image. The program also generated a histogram of the distribution of pixel intensity in the captured image for red, green, and blue. Data were transferred to a spreadsheet program for analysis.

Average values for blue pixel intensity for the entire image of each sheet in the calibration set and light readings from the quantum sensor were used to develop an equation to convert blue pixel intensity values to irradiance in $\mu \mathrm{mol} \cdot \mathrm{s}^{-1} \cdot \mathrm{m}^{-2}$ (Fig. 1). For the canopy comparison, individual histograms were smoothed using a running mean of four values before averaging and statistical analysis. Canopy numerical data was compared using a two-tailed paired t test.

\section{Results and Discussion}

Photosensitivepaper. The Sunprint paper used in this study is one of several products available for making permanent shadow images. The advantage of this type of paper is its ready availability and ease of development. Exposed papers are fixed and developed in water. The ozalid paper used in earlier studies must be developed in a chamber with ammonia gas.

Calibration. The blue pixel value in the captured images had a strong negative correlation with the log of the incident irradiance ( $r=-0.965$ ) (Fig. 1). A similar log relationship between paper sensitivity and light was found in other studies of photosensitive paper (Emmingham and Waring, 1973; Friend, 1969). Red, green, total intensity, and various mathematical combinations of blue, red, green, and total pixel intensity gave significantly poorer correlations (data not shown). Blue pixel value is the dependent variable in this regression. It has been placed on the $\mathrm{x}$ axis in this figure, however, as a predictor of irradiance. The regression equation shown was used to convert blue pixel value to irradiance throughout this paper.

To find a full range of light levels for this calibration, we used a variety of different plant canopies. In retrospect, it may have been better to use only grape canopies for the shaded readings in the calibration set. Canopies of plant species have been reported to have differing effects on light quality and on the calibration curve for ozalid paper, particularly in low, diffuse light (Federer and Tanner, 1966). The narrow spectral response range of photochemical methods of measuring light levels have been a subject of criticism (Pearcy, 1989). However, the calibration curve in Fig. 1 showed that the response of the photosensitive paper and a quantum sensor were closely related in a range of direct and indirect lighting conditions. Where measurement of a specific spectral response is desired, calibration with a spectral radiometer might be more appropriate.

The lighting conditions during video image capture were particularly important. The best resolution across the full range of exposure conditions was achieved when the light levels during the video capture were adjusted by varying the height of the light source to maximize the visible differences in the darkest papers (most sun-exposed). It was essential that the light set up for video capture was identical for the calibration set and the papers exposed in the grape canopies.

Single cluster analysis. A unique aspect of this method is that the incident light on individual paper tubes can be described in significant detail. A selection of exposed papers from the canopy experiment is shown in Fig. 2. Exposed papers from both canopies developed a range of patterns. Papers from shaded cluster positions appear white, whereas those from highly exposed positions have a dark band from the most-exposed side of the tube. The
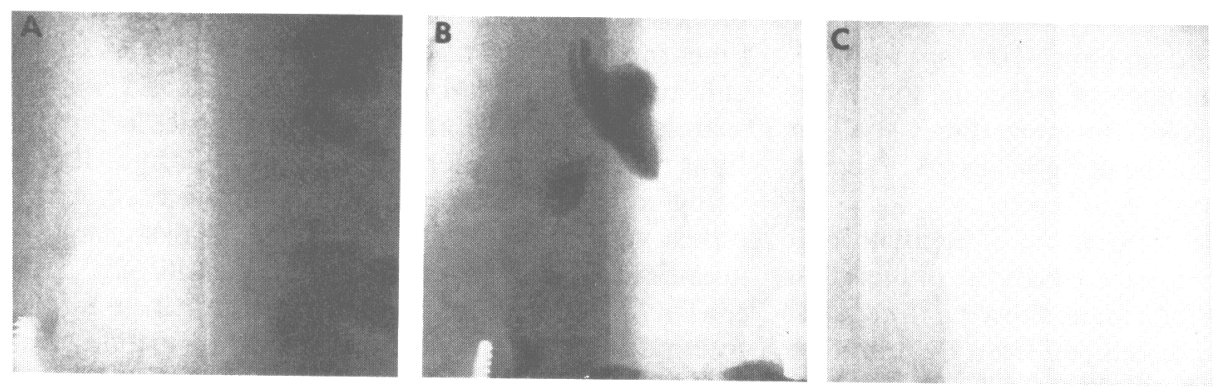

Fig. 3. Photosensitive papers representmg three cluster light exposure situations. (A) A well exposed cluster on the top of a canopy, (B) a moderately exposed cluster with sunflecks, and $(\mathbf{C})$ a shaded cluster. Faint leaf shadows are visible in the dark section of $\mathrm{A}$ and dark spots resulting from sunflecks are clearly visible in B. 

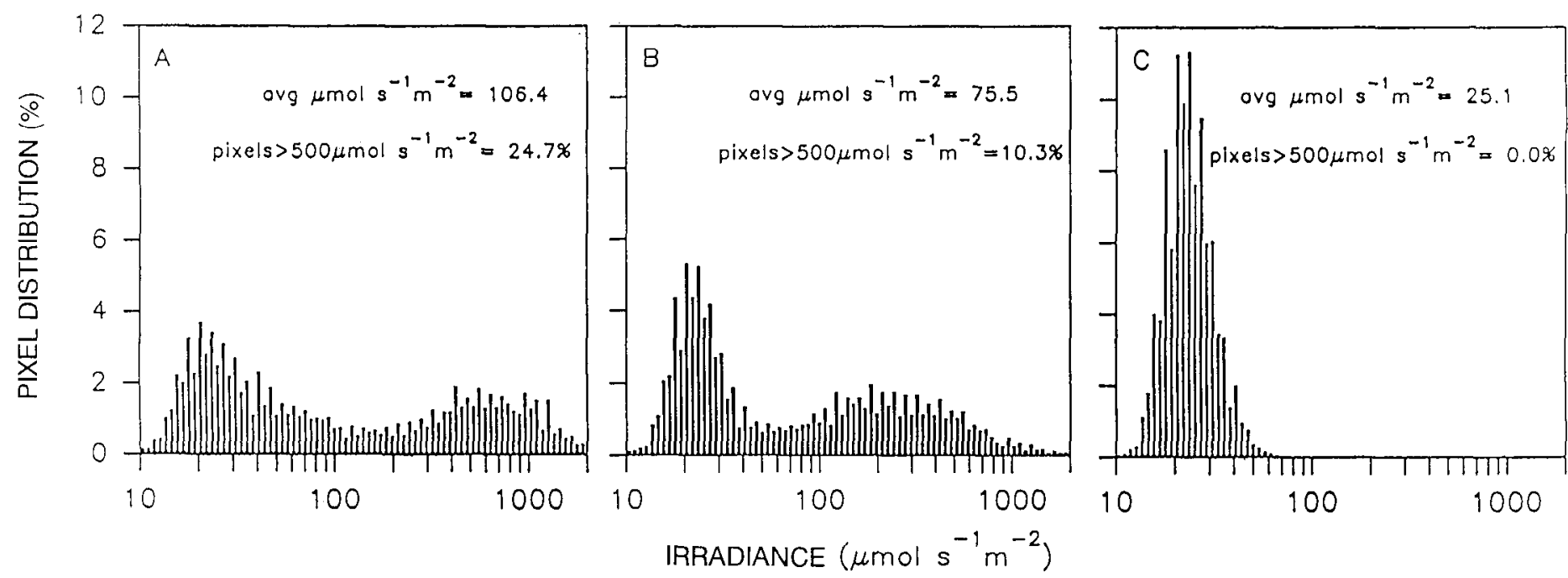

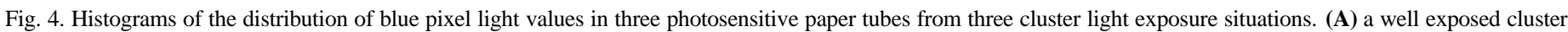
on the top of a canopy, (B) a moderately exposed cluster with sunflecks, and (C) a shaded cluster. The height of each point is the percent of the total pixels at that light level. The area of each curve is the same an is equal to $100 \%$.

effects of sunflecks are readily apparent in many of the papers as dark spots. The striking patterns on the exposed tubes can be used to demonstrate canopy effects on cluster light exposure. We have used these papers in extension demonstrations on canopy management, much as water-sensitive paper is used to demonstrate sprayer effectiveness.

Three exposed papers from very different cluster positions are presented in Fig. 3 to illustrate the information available from individual exposures. Paper A was in one of the most-sun-exposed positions near the top of the minimally pruned canopy. It has a dark, sun-exposed band that faced the sky and a white area that faced the interior of the canopy. Paper B was from a moderately exposed position from the upright trellis. It was exposed to indirect sky light with one prominent dark spot from a sunfleck. Paper C was from a shaded position in the upright trellis with no direct sunlight and very little indirect light.

The distinct distribution pattern of blue pixels in the three exposures is illustrated in the histograms (Fig. 4 A-C). Individual cluster histograms are shown without smoothing. The letter designation for each histogram corresponds to the photo in Fig. 3. The value of the $\mathrm{x}$ axis of each histogram is derived from the blue pixel value using the regression in Fig. 1. The $\mathrm{x}$ axis is in log scale to keep the histogram bin spacings equal (blue pixel value is related to the $\log$ of irradiance) and to differentiate more clearly the light distribution at low light levels $\left(<100 \mu \mathrm{mol} \cdot \mathrm{s}^{-1} \cdot \mathrm{m}^{-2}\right)$. The height of each bar in the histogram is equal to the percent of pixels found at that light intensity level, and the total area of each histogram is the same and equal to $100 \%$. Average light levels for the entire image and the percent of pixels with a light value $>500 \mu \mathrm{mol} \cdot \mathrm{s}^{-1} \cdot \mathrm{m}^{-2}$ are shown at the top of each histogram.

Paper C, from the shaded position, clearly was exposed to the lowest overall light level and received no light $>100 \mu \mathrm{mol} \cdot \mathrm{s}^{-1} \cdot \mathrm{m}^{-2}$. The other two papers are similar, but paper A, from the top of the minimally pruned canopy, was exposed to more direct sunlight $\left(>500 \mu \mathrm{mol} \cdot \mathrm{s}^{-1} \cdot \mathrm{m}^{-2}\right)$ than paper B from the upright canopy. Paper A was also exposed to the highest average light levels.

The histograms used to show the distribution of incident light on clusters are only one way to use the information contained in the image on the exposed tubes. Surface maps showing the spatial distribution of incident light can be developed from a calibration curve like that in Fig. 1 and acaptured video image. Contour maps, wire frame maps, or false color images can all be developed from the database of the captured image.

Canopy differences. A population curve of pixel distribution was assembled from histograms of the 20 paper tubes exposed in each canopy (Fig. 5). The standard errors at the top of the figure are for the smoothed data. Tubes from the minimal pruning canopy had a greater proportion of their surface area exposed to light levels $>50 \mu \mathrm{mol} \cdot \mathrm{s}^{-1} \cdot \mathrm{m}^{-2}$ (Fig. 5). Average irradiance incident on typical cluster positions in the minimally pruned canopy was $65 \%$ higher than the level of the upright canopy (Table 1). Images of tubes from the minimally pruned canopy had significantly greater percentage of pixels at light values above thresholds $>50$ and $100 \mu \mathrm{mol} \cdot \mathrm{s}^{-1} \cdot \mathrm{m}^{-2}$ than the upright canopy but not $>500 \mu \mathrm{mol} \cdot \mathrm{s}^{-1} \cdot \mathrm{m}^{-2}(\mathrm{p}=0.077)$ (Table 1).

These results agree with visual observations of the two canopies. The canopies used for this study were deliberately chosen for their apparent large differences in cluster exposure. The timing of the study (midday) was also chosen to maximize cluster sun exposure differences. Clusters in an upright vertical canopy with a north to south row orientation received almost no direct sunlight near solar noon because of shading from the upper part of the canopy. The differences would have been less pronounced in early morning or late afternoon. Placing tubes in the same cluster positions morning, noon, and afternoon and at different times during the season should be investigated to determine their usefulness in describing season-long effects on cluster exposure. Placing larger tubes of photosensitive paper over a cluster rather than near the cluster may increase the accuracy in describing cluster light exposure.

Field exposure of the photosensitive tubes is a relatively quick procedure. In this experiment, two people were able to expose 40 tubes in $25 \mathrm{~min}$. Additional time was required for establishing the calibration curve and in image capture and analysis. Field research time is often at a premium, particularly under changing light conditions. Determining only four light levels per cluster using a spot quantum sensor would take significantly longer. unless very short integration times were used, and would result in significantly less information on incident light.

The 5-min exposure of the photosensitive tubes appeared to integrate most of the short-term variation in light caused by windinduced movement of sun flecks. Integration of longer term variation caused by diurnal changes in the sun's position was not 


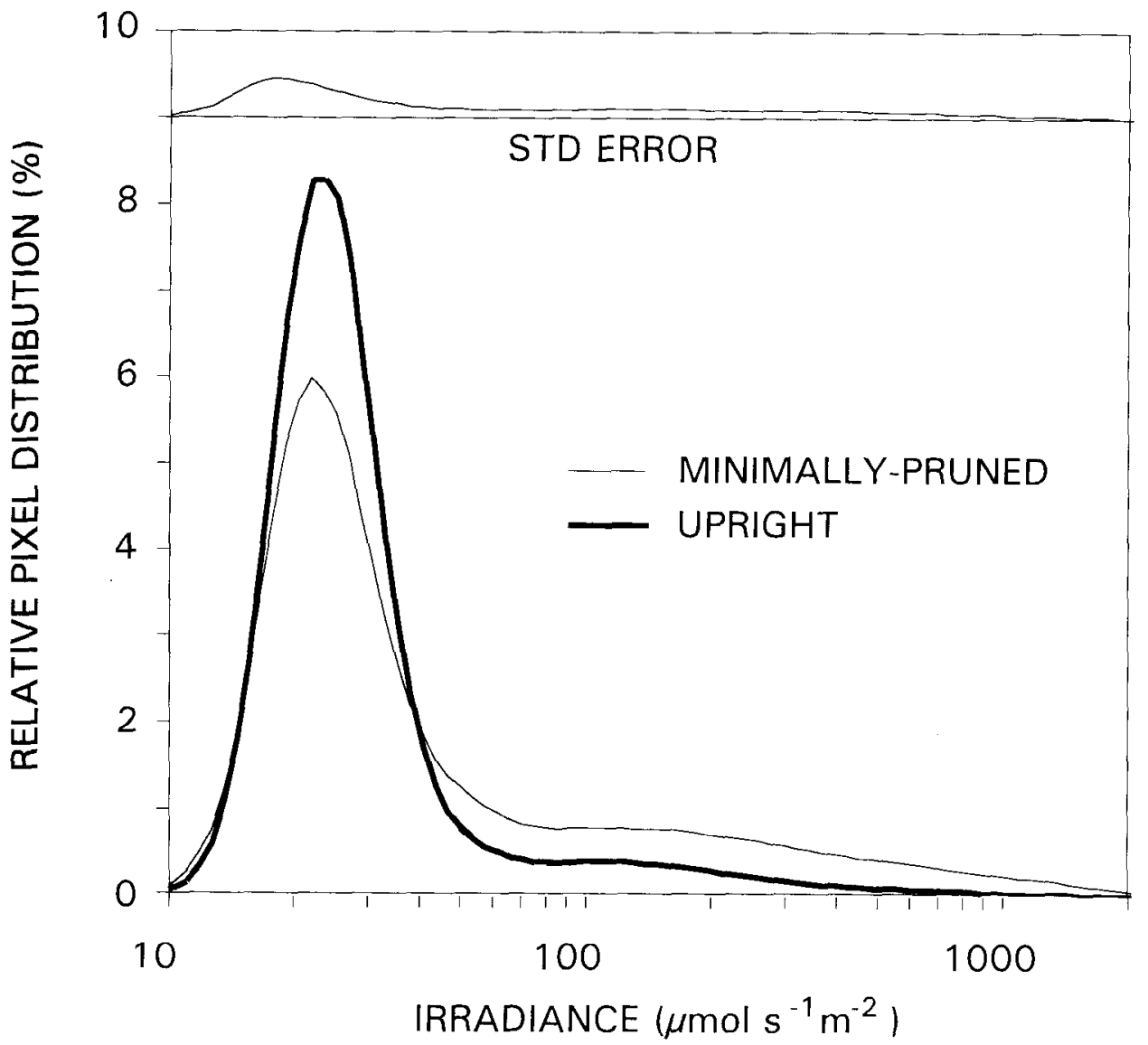

Fig. 5. Population distribution of pixel light values in photosensitive paper tubes from typical cluster positions from two grape canopies: an upright vertical canopy with clusters at the base of the canopy and a minimally pruned canopy with clusters on the periphery of the canopy. The area of each curve is the same. Data was smoothed with a four number running mean. The width of the standard error curve corresponds to the standard error at each point on the plot.

possible with this system. Either multiple observations would need to be taken as described above, or the sensitivity of the paper would need to be changed through the use of filters or different types of photosensitive paper.

\section{Conclusion}

Equipment for digital analysis of video images is becoming more available to researchers in the plant sciences. The detailed images recorded on photosensitive paper coupled with the wide range of analytical options make this an intriguing technique for analysis of light environments. The tubes used in this study were a useful approximation of the light-absorbing surface of a cylindri-

Table 1. Average irradiance and distribution of irradiance in photosensitive paper tubes from typical cluster positions from two grape canopies: an upright vertical canopy with clusters at the base of the canopy and a minimally pruned canopy with clusters on the periphery of the canopy. $P$ values were derived from a two-tailed paired $t$ test.

\begin{tabular}{cccc}
\hline \hline & \multicolumn{2}{c}{ Canopy } \\
\cline { 2 - 3 } & Upright & Minimal & $P$ \\
\hline $\begin{array}{c}\text { Average irradiance } \\
\left(\mathrm{m} \mathrm{mol} \cdot \mathrm{s}^{-1} \cdot \mathrm{m}^{-2}\right)\end{array}$ & 26.7 & 44.2 & 0.025 \\
\multicolumn{5}{l}{ Pixel distribution of irradiance $(\%)$} & & \\
$>50 \mathrm{~m} \mathrm{~mol} \cdot \mathrm{s}^{-1} \cdot \mathrm{m}^{-2}$ & 11.1 & 27.7 & 0.011 \\
$>100 \mathrm{mmol} \cdot \mathrm{s}^{-1} \cdot \mathrm{m}^{-2}$ & 6.2 & 18.3 & 0.018 \\
$>500 \mathrm{mmol} \cdot \mathrm{s}^{-1} \cdot \mathrm{m}^{-2}$ & 0.8 & 4.1 & 0.077 \\
\hline
\end{tabular}

cal grape cluster. The detailed information on the distribution of incident light on the cluster surface would have been difficult to obtain from other light-recording instruments. We are continuing to evaluate the system as a method of predicting flavonol responses in grape skins, but it would appear that the general concept could be easily applied to a wide range of light studies, particularly where the form of the paper can be easily shaped to the measured surface, such as in studies on leaves. The problem of the spectral response of photosensitive papers may be a significant concern, particularly relative to photosynthetic response, but careful calibration and additional research could address that problem.

\section{Literature Cited}

Brodenfeldt, R. and H. Mohr. 1988. Time courses for phytochromeinduced enzyme levels in phenylpropanoid metabolism (phenylalanine ammonia-lyase, naringenin-chalcone synthase) compared with time courses for phytochrome-mediated end-product accumulation (anthocyanin, quercetin). Planta 176:383-390.

Clingleffer, P.R. 1984. Production and growth of minimal pruned Sultana vines. Vitis 23:42-54.

Emmingham, W.H. and R.H. Waring. 1973. Conifer growth under different light environments in the Siskiyou mountains of southwestern Oregon. Northwest Sci. 47:88-98.

Federer, CA. and C.B. Tanner. 1966. Spectral distribution of light in the forest. Ecology 47:555-560.

Friend, D.T.C. 1969. A simple method of measuring integrated light values in the field. Ecol. 42:577-580.

Gutshick, V.P., M.H. Barron, D.A. Waechter, and M.A. Wolf. 1985. Portable monitor for solar radiation that accumulates irradiance histograms for 32 leaf-mounted sensors. Agri. For. Meteorol. 33:281-290. Pearcy, R.W. 1989. Radiation and light measurements, p. 97-116. In: 
R.W. Pearcy, J.R. Ehleringer, H.A. Mooney. and P.W. Rundel (eds.). Plant physiological ecology. Chapman and Hall, New York.

Price, S.F., P.J. Breen, M. Valladao, and B.T. Watson. 1994. Cluster sun exposure and quercetin in grapes and wine. Amer. J. Enol. Viticult. 46. (In press.)

Price, S.F., B.J. Yoder, P.J. Breen, and B.T. Watson. 1992. Solarradiation effects on anthocyanins and phenolics in skins of Pinot noir and Pinot gris, p. 563-570. In: Proc. 4th Intl. Symp. Grapevine Phys., Torino, Italy, May, 1992.

Reifsnyder, W.E., G.M. Furnival, and J.L. Horowitz. 1971. Spatial and temporal distribution of solar radiation beneath forest canopies. Agri. Meteorol. 9:21-37.

Reynolds, A.G. and D.A. Wardle. 1989a. Effects of timing and severity of summer hedging on growth, yield, fruit composition. and canopy characteristics of de Chaunac. I. Canopy characteristics and growth parameters. Amer. J. Enol. Viticult. 40: 109-120.
Reynolds. A.G. and D.A. Wardle. 1989b. Influence of fruit microclimate on monoterpence levels of Gewürztraminer. Amer. J. Enol. Viticult. 40:149-154.

Shaulis, N.. H. Amberg, and D. Crowe. 1966. Response of Concord grapes to light exposure and Geneva double curtain training. Proc. Amcr Soc. Hort. Sci. 89:268-280.

Smart, R.E. 1982. Vinemanipulation toimprove winegrape quality. Proc. Symp. Grape and Wine Cent. June 1980. A.D. Webb (ed.) (Univ. Calif.. Davis) pp 362-375.

Smart, R.E. 1985. Principles of grapevine canopy microclimate manipulation with implications for yield and quality. A review. Amer. J. Enol. Viticult. 36:230-239.

Smart. R.E. 1988. Shoot spacing and canopy light microclimate. Amer. J. Enol. Viticult. 39:325-333.

Stafford. H.A. 1990. Flavonoid metabolism. CRC Press. Boca Raton. Fla. 